\title{
MDR TUBERCULOSIS - A GLOBAL THREAT
}

\author{
Maruti Sawadkar \\ Department of Pharmacology \\ Universal College of Medical Sciences \\ Bhairahawa, Nepal
}

Though tuberculosis (TB) is the oldest disease, yet it remains to be one of the top 10 causes of death worldwide. The lack of change in the treatment of TB over the past four decades and failure of Directly Observed Treatment, Short-course (DOTS) has resulted in development of strains resistant to multiple first line drugs. This has resulted in rise of Multi drug resistant tuberculosis (MDR TB) and Extensive drug resistant tuberculosis (XDR TB) posing a global threat to human life. ${ }^{1}$ MDR TB is one of the major cause of global deaths. About 600,000 new cases of MDR-TB emerge each year and about 240,000 of these people die. ${ }^{2}$

According to World Health Organization (WHO), MDR TB is a resistant form of $\mathrm{TB}$ at least to isoniazid and rifampicin whereas XDR TB is a form of TB resistant to at least four of the core anti-TB drugs and also to any of the fluoroquinolones (such as levofloxacin or moxifloxacin) and to at least one of the three injectable second-line drugs (amikacin, capreomycin or kanamycin).

Two years back, with so-called greater collaborations and research, WHO had claimed a significant progress in the treatment of MDR and XDR tuberculosis and had put antituberculosis drugs for MDR-TB and XDR into 4 major groups. ${ }^{4}$

The list of the drugs (Tables 1 and 2) published by them, ${ }^{4}$ was nothing but re-arrangement of the same old drugs which were once put in the list of second line drugs because of their less efficacy and more toxicity or had very minimum anti TB activity.

\section{Table 1. Showing group $B$ and $C$ drugs}

\begin{tabular}{|c|c|}
\hline $\begin{array}{l}\text { Group B: } \\
\text { Second-line injectable } \\
\text { agents }\end{array}$ & $\begin{array}{l}\text { Group C: } \\
\text { Other core second-line } \\
\text { agents }\end{array}$ \\
\hline $\begin{array}{l}\text { 1. Amikacin } \\
\text { 2. Capreomycin } \\
\text { 3. Kanamycin } \\
\text { 4. Streptomycin }\end{array}$ & $\begin{array}{l}\text { 1. Ethionamide/prothionamide } \\
\text { 2. Cycloserine/terizidone } \\
\text { 3. Linezolid } \\
\text { 4. Clofazimine }\end{array}$ \\
\hline
\end{tabular}

Table 2. Showing group D1 and D3 drugs

\begin{tabular}{|l|l|}
\hline Sub group D1 & Sub group D3 \\
\hline 1.Pyrazinamide & 1. Para-aminosalicylic acid \\
2.Ethambutol & 2. Imipenem plus Cilastatin \\
3. High-dose & 3. Meropenem \\
isoniazid & 4. Amoxicillin plus Clavulanate \\
& 5. Thioacetazone \\
\hline
\end{tabular}

Their treatment strategy also had shown more hope in group A drugs (Table 3) which includes fluoroquinolones and two new drugs in sub group D2, bedaquiline and delamanid which were still under clinical trail.

\section{Table 3. Showing group A and D2 drugs}

\begin{tabular}{|l|l|}
\hline Group A: fluoroquinolones & Sub group D2 \\
\hline 1. Levofloxacin & 1. Bedaquiline \\
2. Moxifloxacin & 2. Delamanid \\
3. Gatifloxacin & \\
\hline
\end{tabular}

Repurposed drugs licensed for other conditions were also recommended in the interim period ${ }^{5-7}$ till new drug regimen is available.

The previous guidelines by WHO recommended that during the intensive phase patients with MDR-TB not eligible for the shorter regimen should receive at least five active anti-TB medicines.

Along with pyrazinamide they should receive:

- One drug from group A

- One drug from group B

- Two drugs from group C

According to WHO, if after drug sensitivity report, the minimum of effective anti-TB drugs cannot be composed from above groups, one agent from group D2 and other agents from D3 may be added to bring the total to five.

Realising fragile progress in the treatment of MDR-TB due to this strategy, recently in their rapid communication (August 2018), WHO came out with new strategy for the treatment of MDR-TB which includes all three drugs - levofloxacin or moxifloxacin, bedaquiline ${ }^{89}$ and linezolid from group A; two drugs - clofazimine and cycloserine or terizidone from group $\mathrm{B}$ as the drugs of first choice unless they cannot be used due to resistance or severe side effects.

Under such situation, depending up on their potency, toxicity and drug resistance, rest of the drugs such as ethambutol, delamanid, ${ }^{10}$ imipenem, pyrazinamide, cilastatin or meropenem, amikacin or streptomycin, ethionamide or prothionamide and $\mathrm{p}$-aminosalicylic acid from group $\mathrm{C}$ were recommended. ${ }^{11}$

DOI: https://doi.org/10.3126/jucms.v6i2.22466 
As compared to previous guidelines the treatment suggested in August 2018 guidelines is simple and easy to implement.

However, development of this regimen combining new and existing drugs, recommended by WHO requires detailed information on their respective sensitivity, safety and toxicity. In this therapy, their potential for drug-drug interactions when used in HIV and their propensity for development of drug toxicity while on therapy in pregnant women, and children must be also considered.

Previous treatment courses for drug-resistant tuberculosis was ambiguous. Although, WHO supported the use of the ninemonth "Bangladesh" regimen, many patients did not meet the eligibility criteria, and needed therapy of 18 months' duration or more. $^{12-14}$

Thus compared with treatment of drug-susceptible TB, treatment of MDR-TB requires longer course duration and more expensive drugs with a higher toxicity profile. The requirement of several antimicrobials and the extended treatment period complicate the ability to administer, manage and follow patients, who frequently are not able to comply or tolerate treatment. Moreover, the accessibility of drug susceptibility testing, therapeutic drug monitoring and thus effective treatment regimens for MDR and XDR-TB further complicate matter.

Drug resistant tuberculosis is a significant challenge for the control of TB in many parts of the world and a threat to TB elimination program. The origin of this problem has been the sub-optimal management of patients with susceptible TB. Inadequate management of cases can be the origin of $50 \%$ of the new MDR-TB cases. The other $50 \%$ is due to active transmission of MDR-TB strains in the community or healthcare settings.

Therefore, to control this epidemic, we need to improve the management of the susceptible TB cases as well as find and cure most of the MDR- TB cases, to whom best treatment options must be assured.

It is the need of present situation, that not to repeat previous errors with these drugs, make these drug available easily free of cost, monitor drug sensitivity, identify and manage drug interaction and drug toxicity; as patient is treated with nearly five drugs and to develop a universal new regimen with these new drugs for all TB patients, susceptible and resistant to all the old drugs.

\section{REFERENCES}

1. World Health Organization. Global tuberculosis control: WHO report 2000. Geneva: World Health Organization; 2000. WHO document $\mathrm{WHO} / \mathrm{CDS} / \mathrm{TB} / 2000.275$.

2 World Health Organization WHO treatment guidelines for drug-resistant tuberculosis. 2016 update. WHO/HTM/ TB 2016. 04 World Health Organization, Geneva (2016).
3 World Health Organization The End TB Strategy. Global strategy and targets for tuberculosis prevention, care and controla, after 2015 .

4 WHO treatment guidelines for Refampicin and multidrug resistant tuberculosis, 2018 update.

5 R.S. Wallis, M. Maeurer, P. Mwaba, J. Chakaya, R.Rustomjee, G.B. MiglioriTuberculosisadvances in development of new drugs, treatment regimens, host-directed therapies, and biomarkers. Lancet Infect Dis, 16 (2016), pp. e34-e46.

6 D. Falzon, H.J. Schünemann, E. Harausz, et al. WHO treatment guidelines for drug-resistant tuberculosis, 2016 update Eur Respir J, 49 (2017) article 1602308.

7 Target regimen profiles for TB treatment: candidates: Rifampicin-susceptible, Rifampicin-resistant and pan-TB treatment regimens. WHO Library Cataloguing-in Publication Data.

8 Moloko C. Cholo Maborwa T. Mothiba Bernard, Fourie Ronald Anderson: Mechanisms of action and therapeutic efficacies of the lipophilic antimycobacterial agents Clofazimine and Bedaquiline Journal of Antimicrobial Chemotherapy, Volume 72, Issue 2, 1 February 2017, Pages 338-353.

9 L. Guglielmetti, D. Le Dû, N. Veziris, E. Caumes, D. MarigotOuttandy, Y. Yazdanpanah, et al.Is bedaquiline as effective as fluoroquinolones in the treatment of multidrug-resistant tuberculosis? Eur Respir J, 48 (2016), pp. 582-585.

10 Alphienes Stanley Xavier and Mageshwaran Lakshmanan Delamanid: A new Armor in combating drug-resistant tuberculosis: J Pharmacol Pharmacother. 2014 Jul-Sep; 5(3): 222-224.

11. Rapid Communication: Key changes to treatment of multidrug and rifampicin-resistant tuberculosis (MDR/RRTB) August 2018

12 G. Sotgiu, S. Tiberi, L. D'Ambrosio, R. Centis, J.W. Alffenaar, J.A. Caminero Faster: The new 'shorter' regimen for multidrugresistant tuberculosis Eur Respir J, 48 (2016), pp. 1503-1507 CrossRefView Record in Scopus.

13 WHO factsheet: The shorter MDR-TB regimen (2016) Availablefrom: http://www.who.int/tb/Short_MDR_ regimen_factsheet.pdf.

14 M. Dalcolmo, R. Gayoso, G. Sotgiu, L. D'Ambrosio, J.L. Rocha, L. Borga, et al.Resistance: Profile of drugs composing the "shorter" regimen for multidrug-resistant tuberculosis in Brazil, 20002015 Eur Respir J, 49 (2017). 